\section{An miRNA boost for p53}

\section{By Kai-Jye Lou, Staff Writer}

Although many studies have shown that altered expression of one or more microRNAs is associated with cancer, they have not shown that the miRNAs could be therapeutically targeted with a drug-like molecule. Now, U.S. and Australian researchers have shown for the first time in vivo that blocking miRNA can slow tumor growth.

"For years, there have been studies suggesting that the $3^{\prime}$ untranslated region of $p 53$ mRNA could be important for the regulation of the protein," said Andrei Goga, assistant professor in the Department of Medicine at the University of California, San Francisco. "What we have been trying to do is to identify microRNA sequences that target this region."

Goga is a corresponding author on the paper, which was published in Nature Medicine. Alex-
"This is the first report showing that small drug-like microRNA antagonists can diminish tumor growth in a whole animal."

-Andrei Goga, University of California, San Francisco
"We found that small oligonucleotide inhibitors can block the activity of miR-380-5p and increase p53, thus leading to the death of neuroblastoma cells and decreased tumor growth in a whole mouse," said Goga. "This is the first report showing that small druglike microRNA antagonists can diminish tumor growth in a whole animal. We hope this will pave the way for developing these and other microRNA antagonists as anticancer therapies."

"The work provides further support to the idea that microRNAs are well-positioned to be good targets in oncology," said Eric Marcusson, a coauthor on the paper and director of oncology at Regulus Therapeutics Inc. "The current study shows that we are able to deliver the microRNA antagonists in a manner that is therapeutically relevant and has an antitumor effect. Other researchers have not yet done this at the whole-animal level, though there may have been some preliminary demonstrations in cell culture."

Regulus developed the chemically modified anti-miR-380-5p oligonucleotides used in the mouse tumor inhibition study, although the company currently is pursuing other miRNA targets: it has antagonists of miR-21 and liposomeencapsulated miR-34 mimics in preclinical development for cancer.

Studies from other labs have reported that two other miRNAs-miR-125b and miR504-are negative regulators of $\mathrm{p} 53$, but they used research reagents to antagonize miRNA ander Swarbrick and Susan Woods were the co-lead authors. Swarbrick is a senior research officer and leader of the Tumour Progression Group at the Garvan Institute, and Woods is a research officer in the Oncogenomics Laboratory at the Queensland Institute of Medical Research.

Initially, the group identified miR-380-5p as an oncogene that repressed tumor protein p53 (TP53; p53) and blocked senescence and apoptosis, thus showing that there is a parallel pathway for p53 regulation alongside the better-known MDM2 p53 binding protein homolog (MDM2; HDM2) pathway. Of 205 primary patient neuroblastoma samples, 203 had detectable miR-380-5p expression and 155 had overexpression compared with levels in normal brain tissue.

"The work identifies a parallel pathway for p53 regulation, in addition to MDM2, whereby p53 function is attenuated," said Joshua Mendell, an associate professor of pediatrics, molecular biology and genetics at The Johns Hopkins University. "The findings certainly raise the possibility that in tumor types where p53 is still functional, this microRNA could be a therapeutic target."

MDM2 is a negative regulator of p53. At least five companies are developing compounds that inhibit MDM2. The two most advanced candidates are in Phase I testing for various cancers: R7112 from Roche and JNJ-26854165 from Johnson \& Johnson.

In the current work, using a mouse model of neuroblastoma, twiceweekly injections with chemically modified antisense oligonucleotides targeting miR-380-5p significantly decreased tumor size and weight compared with injections of control oligonucleotides $(p=0.01) .{ }^{1}$ activity, which are not therapeutically tractable., ${ }^{2,3}$

"Many cancers show a loss of p53 activity and/or function, and there are multiple mechanisms that lead to defective p53," said Andreas Bader, associate director of research at Mirna Therapeutics Inc. "However, only when we know all the different mechanisms of how cancer genes are regulated can we generate appropriate tools to inhibit cancer. This work is a valuable contribution in that direction and suggests that an anti-miR-380-5p can be a promising therapeutic approach for cancers that show wild-type p53 and elevated levels of miR-380-5p."

In June, Bader's group at Mirna showed the flip side of what the current paper shows: that both local and systemic delivery of a miR34 a mimic could block tumor growth in mouse models of non-small cell lung cancer (NSCLC). ${ }^{4,5} \mathrm{miR}-34 \mathrm{a}$ is activated by p53 and has tumor suppressor activity (see Figure 1, “Targeting microRNAs in the p53 signaling pathway").

Mirna has miR-34 mimics in preclinical development for solid cancers.

\section{Form and function}

Neuroblastomas were a good test case for confirming the role of miR$380-5 p$ in regulating p53 because the miRNA is upregulated in these cancers, which also retain wild-type p53. Having functional p53 was important because inhibiting miR-380-5p failed to induce senescence and cell death in the absence of $\mathrm{p} 53$. 
Figure 1. Targeting microRNAs in the p53 signaling pathway. In normal cells, oncogenic signals like stress and DNA damage activate the tumor protein p53 (TP53; p53), which then activates cellular pathways involved in apoptosis, cell-cycle arrest and senescence. p53 signaling is downregulated in multiple cancers, and restoring its function can have an anticancer effect.

In Nature Medicine, U.S. and Australian researchers identified microRNA-380-5p (miR-380-5p) as a negative regulator of $p 53$ and showed that this miRNA is an oncogene that is upregulated in most neuroblastomas. The researchers also showed that systemic delivery of an anti-miR-380 provided by Regulus Therapeutics Inc. increases p53 activity and decreases the growth of transplanted primary neuroblastoma tumors in mice [a].

Earlier studies also have identified miR-125b and miR-504 as negative regulators of p53 that can drive various cancers [b]. In addition, p53 also is known to activate the family of miR-34s, which can act as tumor suppressors that block the expression of multiple downstream targets implicated in cancer.

Regulus and Mirna Therapeutics Inc. both have miR-34 mimics in preclinical development for various cancers [c].

"There has been a lot of interest over the years on identifying strategies to reactivate p53," said Goga. "This work shows that there is potentially another way to upregulate this tumor suppressor. The advantage with this microRNA is that it does not appear to be widely expressed in adult tissues but is found in nearly all neuroblastomas. Thus, antagonizing this microRNA could specifically upregulate p53 in tumor cells and induce cell death."

"An anti-miR-380-5p-based therapy may well complement existing therapies, as already suggested by the authors, and also may be a valuable agent in treating neuroblastomas that have become resistant due to downregulation of 53 by miR-380-5p," Bader told SciBX.

Mendell said the real challenge will be to develop an efficient delivery method for the miRNA antagonists. In addition, he thinks it will be important to search for other proteins that could be regulated by miR-380-5p. "The mechanism of action for microRNAs is still not wellestablished, and they could be acting on multiple targets," he noted.

Goga agreed that miR-380-5p could be regulating other proteins but said the antitumor effect from miR-380-5p antagonism is dependent on $\mathrm{p} 53$.

Marcusson said it would be important to test the relevance of miR380-5p expression in other models of neuroblastoma as well as exploring its role in other tumor types. "Some preliminary data have suggested that this microRNA could also play a role in other cancer types," he said.

Indeed, Goga said the group is working to identify other cancers in which antagonists of miR-380-5p could have utility.

"This microRNA is not widely expressed-it's seen primarily in cells differentiated along the neural crest lineage," he told SciBX. "For the moment we are looking at melanoma, which also is derived from the
miR-34 mimics in preclinical development for solid cancers from Mirna Therapeutics Inc.;

Anti-miR-380-5p from Regulus Therapeutics Inc.

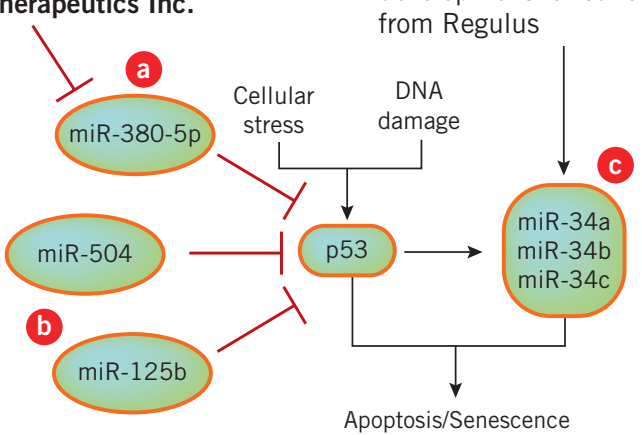

neural crest lineage. We are also looking at the possibility of targeting the microRNA in other cancers, such as liver cancers-as the small nucleic acid-based antagonist can get into this tissue quite readily."

The findings published in Nature Medicine are not patented. Zachary Zimmerman, director of business development at Regulus, said the company holds multiple patents covering miRNA-based therapeutics including composition of matter, therapeutic methods of use and chemical modifications for miR-380-5p and numerous other miRNAs.

Lou, K.-J. SciBX 3(39); doi:10.1038/scibx.2010.1169

Published online Oct. 7, 2010

\section{REFERENCES}

1. Swarbrick, A. et al. Nat. Med.; published online Sept. 21, 2010; doi:10.1038/nm.2227

Contact: Andrei Goga, University of California, San Francisco, Calif. e-mail: andrei.goga@ucsf.edu

Contact: Alexander Swarbrick, Garvan Institute, Sydney,

New South Wales, Australia

e-mail: a.swarbrick@garvan.org.au

2. Le, M.T.N. et al. Genes Dev. 23, 862-876 (2009)

3. Hu, W. et al. Mol. Cell 38, 689-699 (2010)

4. Wiggins, J.F. et al. Cancer Res. 70, 5923-5930 (2010)

5. Fulmer, T. SciBX 3(28); doi:10.1038/scibx.2010.848

\section{COMPANIES AND INSTITUTIONS MENTIONED}

Garvan Institute, Sydney, New South Wales, Australia The Johns Hopkins University, Baltimore, Md.

Johnson \& Johnson (NYSE:JNJ), New Brunswick, N.J. Mirna Therapeutics Inc., Austin, Texas

Queensland Institute of Medical Research, Brisbane, Queensland, Australia

Regulus Therapeutics Inc., Carlsbad, Calif.

Roche (SIX:ROG; OTCQX:RHHBY), Basel, Switzerland

University of California, San Francisco, Calif. 